\title{
The Practice of Constraint in Psychiatry: Emergent Forms of Care and Control
}

\author{
Paul Brodwin • Livia Velpry
}

Published online: 26 September 2014

(C) Springer Science+Business Media New York 2014

The work of psychiatry has always revolved around the twin imperatives of care and custody. In an older model of psychiatric power, confinement and coercion were accomplished through isolation from society. The asylum became the ultimate symbol of such power. It arose in the long historical process Foucault termed "the great confinement," and it reached its ultimate expression in the "total institution" exposed by Goffman in the mid-20th Century. As the landscape of mental health shifted, anthropological attention turned away from the asylum to focus on other topics: psychiatry's contribution to social control in the neo-liberal state, as practices of governmentality (Foucault 2012), new technologies of the self (Rose 1996) and pharmaceuticalization and the global health movement (Biehl and João 2011).

Constraint nevertheless remains a common practice even in our post-asylum era. This special section of Culture, Medicine, and Psychiatry examines contemporary tactics of psychiatric constraint through studies of mental health settings in the USA and France. The four papers connect abstract arguments about disorder, ethics, and subjectivity to the rough edge of practice, where the interests of clinicians and patients most sharply diverge. The mandate not only to treat people but also to control them has produced diverse practices of constraint and on-going struggles about their legitimacy. The authors here take up constraint as a therapeutic value, social logic, work routine, and a challenge to the moral self-regard of front-line clinicians.

The four papers illuminate the main contemporary modes of constraint: involuntary commitment, collaboration with criminal courts and prisons, physical restraints, and locked wards. They also reveal the diverse assemblages that enable psychiatric power today. From isolation rooms in hospitals and prisons to forced

\footnotetext{
P. Brodwin (ه)

Milwaukee, USA

e-mail: brodwin@uwm.edu

L. Velpry

Paris, France

e-mail: livia.velpry@univ-paris8.fr
} 
treatment in the community and residential centers for youth, the practice of constraint is always embedded in a larger institutional apparatus. At one level, the warrant for constraint comes from broad notions about mental illness and professional obligations. At another level, however, the warrant comes from the local shape of practice: the particular techniques, rationales, and limits of treatment that differ from site to site and one historical period to the next. Even the words "constraint" and "care" have unstable meanings that depend on national traditions of psychiatry and local ideologies of treatment.

Changes in the regulation of clinical conduct also redefine the frontier between practices that are valued and those considered "dirty work". The line between sanctioned and shameful uses of psychiatric power appears in many different registers: what clinicians say about their face-to-face engagements with patients, how they negotiate the workplace bureaucracy, and much broader societal worries about balancing individual liberty and collective safety. As these papers demonstrate, constraint becomes both a component of care and its ethical limit. The enduring tension between care and control, and the ways it disturbs psychiatric practice at many different scales, demands a multidisciplinary approach. These papers draw on anthropology, sociology, and history to illuminate the strategies and justifications for constraint in particular contexts. They expose the tangled histories lying below today's smooth institutional routines. The papers also delve into the personal experience and self-regard of clinicians who must undergo an "apprenticeship in constraint" (a notion from Henckes's paper). Clinicians must somehow build up a professional persona that allows them to push back against people's strong opposition to forced medications, surveillance, or physical confinement.

The first two papers (by Velpry \& Eyraud and Brodwin) show what is at stake for mental health professionals who navigate the tension between care and control. Are they able to incorporate practices of constraint into their therapeutic models, or at least to give them clinical meaning? Their response shows striking similarities across diverse treatment settings and national contexts. In French high security units and US case management teams, providers are compelled to draw a line between their approach and the work of police and penal officials. Velpry and Eyraud focus on the twin logics of confinement - custody and care, or security and humanity — as these principles structure a range of psychiatric and penal institutions in contemporary France. Their paper provides a detailed history of the prison and the asylum, as well as the blurred boundary separating them. Eyraud and Velpry demonstrate that custody and care exist as a continuum, not a polarity. In the end, the usual administrative logics - from criminal justice and psychiatry-misrecognize the hybrid institutional arrangements where confinement actually takes place, and where deep questions of personal responsibility, collective security, and human dignity remain unsettled. Brodwin explores the ethical sensibility of front-line providers as they oversee physical restraints or collude with the criminal justice system to impose treatment under the threat of imprisonment. Their deep ambivalence about constraint points in two directions at once. It indexes their personal commitment to the humanist ideals pervading contemporary US mental health work. Such ideals coexist uneasily with a therapeutic pragmatism, in which the clinical beneficial consequences of imposed treatment make it ipso facto 
acceptable. In the other direction, their ambivalence points outward to the objective conditions of work in publicly funded community psychiatry. Through the lens of their ambivalence, providers accurately discern the contradictory roles they are forced to play as both carers and punishers, and both friends of clients and watchdogs of their medication adherence. Their personal moral misgivings are thus a barometer of the real contours of psychiatric power.

Papers by Henckes and Hejtmanek explore the dilemmas of front-line providers as they learn to impose limits on their patients. Henckes analyzes a set of 200 selfreflective essays written by young psychiatric nurses-in-training in mid- $20^{\text {th }}$ Century Paris. Their narratives tackle the existential question, who am I to impinge on my patient's will? The question reflects the ethical urgency of the post-war psychiatric reform movement and its explicit valuation of humanistic care. These trainees interrogate their apprenticeship in constraint, their ethical unease and the strategies to contain it, how they learn to justify their action, and how they take up or disavow responsibility for patients' well-being. Hejtmanek examines some of the same quandaries among staff at a contemporary US residential treatment center for youth with serious mental illness. In this setting, staff members are trained to cultivate a particular kind of clinical agency. They must obey the therapeutic ideologies of the center not only to care about and even re-parent the residents, but also to retain a professional distance from the youths' emotional manipulation. It is a delicate balancing act and low-ranking "line staff" constantly chafe under the supervision of credentialed psychologists. Since the staff and residents are mostly African American, and the psychologists mostly white, a racial dynamic drives staff member's discomfort with normative practices of constraint. These two papers show how such practices have a recursive effect on clinicians' self-image, as they find themselves caught up in larger fields of power and control.

\section{References}

Biehl, João

2011 CATKINE ... Asylum, Laboratory, Pharmacy, Pharmacist, I and the Cure: Pharmaceutical Subjectivity in the Global South. In Pharmaceutical Self: the Global Shaping of Experience in an Age of Psychopharmacology. Janis Jenkins, ed., pp. 67-96. Santa Fe, NM: SAR Press.

Foucault, Michel

2012 Du gouvernement des vivants. Cours au Collège de France (1979-1980) Paris: Seuil.

Rose, Nikolas

1996 Inventing Our Selves: Psychology, Power and Personhood. New York: Cambridge University Press. 